\title{
Poda drástica de verão e produção de cultivares de amoreira-preta em região subtropical
}

\author{
Maraisa Hellen Tadeu(1), Filipe Bittencourt Machado de Souza(1), Rafael Pio(1), \\ Matteus Heberth Ribeiro do Valle(1), Guilherme Locatelli(1), Glênia Ferreira Guimarães ${ }^{(1)}$ \\ e Bianca Elis Cruz Silva(1)
}

(1)Universidade Federal de Lavras, Departamento de Agricultura, Caixa Postal 3037, Campus Universitário, CEP $37200-000$ Lavras, MG, Brasil. E-mail: maraisaht@yahoo.com.br, fbmsouza@yahoo.com.br, rafaelpio@dag.ufla.br, matteus_hrv@hotmail.com, guilherme_locatelli_@hotmail.com,gleniaguima@hotmail.com, biancaeliscruzsilva@gmail.com

\begin{abstract}
Resumo - O objetivo deste trabalho foi avaliar o manejo de podas em cultivares de amoreira-preta (Rubus spp.), submetidas à poda drástica de verão, em regiões subtropicais. $\mathrm{O}$ experimento foi conduzido em blocos ao acaso, em arranjo fatorial $2 \times 7$ (podas x cultivares), no Município de Lavras, MG. Foram utilizadas as cultivares Comanche, Caingangue, Choctaw, Tupy, Guarani, Brazos e Cherokee, que receberam poda convencional (controle) e poda drástica de verão. Foram avaliados dados fenológicos, vegetativos e produtivos nos ciclos de produção 2012/2013 e 2013/2014, além de características físico-químicas dos frutos e da incidência de doenças, no último ciclo de produção. A poda drástica de verão aumenta a produção das cultivares em regiões subtropicais. Não há diferença na qualidade dos frutos colhidos de plantas submetidas aos sistemas de poda avaliados. A poda drástica de verão não é eficiente na redução ou no controle de doenças.
\end{abstract}

Termos para indexação: Rubus, fenologia, pequenas frutas, manejo fitossanitário, qualidade de frutos.

\section{Drastic summer pruning and production of blackberry cultivars in subtropical areas}

\begin{abstract}
The objective of this work was to evaluate pruning managements of blackberry (Rubus spp.) cultivars, subjected to drastic summer pruning, in subtropical regions. The experiment was carried out in a randomized complete block design, in a $7 \times 2$ (pruning $\mathrm{x}$ cultivars) factorial arrangement, in the municipality of Lavras, in the state of Minas Gerais, Brazil. The Comanche, Caingangue, Choctaw, Tupy, Guarani, Brazos, and Cherokee cultivars were used, receiving conventional pruning (control) and drastic summer pruning. Phenological, vegetative, and productive data were evaluated on the 2012/2013 and 2013/2014 production cycles, as well as the fruit physicochemical attributes and disease incidence in the last production cycle. Drastic summer pruning increases the production of cultivars in subtropical regions. There is no difference in the quality of fruit harvested from plants subjected to the evaluated pruning systems. Drastic summer pruning is not effective in the reduction or the control of diseases.
\end{abstract}

Index terms: Rubus, phenology, small fruit, phytossanitary management, fruit quality.

\section{Introdução}

$\mathrm{O}$ interesse pelo consumo da amora-preta (Rubus spp.) aumentou nos últimos anos, pelo fato de seus frutos apresentarem quantidades expressivas de compostos, como fenólicos e carotenoides, que podem auxiliar no combate às doenças degenerativas. Também são encontrados, nas amoras-pretas, pigmentos naturais, principalmente a antocianina, que confere uma coloração atraente no processamento e na confecção de produtos lácteos, geleias e doces em caldas (Antunes, 2002; Guedes et al., 2013).
A exploração da amora-preta no Brasil teve início com o lançamento das primeiras cultivares brasileiras, Tupy, Guarani e Caingangue, pelo programa de melhoramento genético da Embrapa Clima Temperado (Figueiredo et al., 2013). Apesar de os primeiros cultivos terem sido introduzidos na região Sul do País (Antunes, 2002; Fachinello et al., 2011), recentemente tem-se observado o aumento das áreas cultivadas com amoreiras-pretas em outros estados, especialmente nas regiões subtropicais do Paraná e de Minas Gerais (Campagnolo \& Pio, 2012b; Guedes et al., 2013). Quanto ao manejo das amoreiras-pretas em regiões 
subtropicais, a alta densidade de espinhos nas hastes dificulta a colheita e as podas realizadas no verão após o término da colheita, uma vez que as elevadas temperaturas dificultam a utilização de vestimentas apropriadas para essas atividades (Campagnolo \& Pio, 2012d).

Recentemente, foi lançada a cultivar Xavante, cuja principal característica é a ausência de espinhos em suas hastes, o que facilita a condução das plantas e, sobretudo, as podas (Fachinello et al., 2011). No entanto, a produção dessa cultivar é baixa em regiões subtropicais. Broetto et al. (2009) registraram produtividade de 3,02 $\mathrm{Mg} \mathrm{ha}^{-1}$ em Guarapuava, PR, enquanto Campagnolo \& Pio (2012b) obtiveram 1,27 $\mathrm{Mg} \mathrm{ha}^{-1}$ em Marechal Cândido Rondon, PR.

A exploração de cultivares adequadas à colheita mecânica, como a Cherokee e a Comanche, ou que apresentem ausência de espinhos em suas hastes, como a Ébano, seria uma opção. Contudo, Antunes et al. (2000) observaram produtividade de apenas 3,26 $\mathrm{Mg}$ ha $^{-1}$ em plantas dessa cultivar em Caldas, MG, bem inferior ao registrado para a cultivar Brazos, que atingiu 16,36 $\mathrm{Mg} \mathrm{ha}^{-1}$. Porém, a cultivar Brazos produz frutos ácidos, impróprios para o consumo ao natural, e apresenta espinhos em alta densidade (Guedes et al., 2013). A cultivar Guarani apresenta boa produtividade, mas seus frutos têm sabor inferior aos da Tupy, que produz frutos com bom equilíbrio entre açúcares e acidez, assim como a Caingangue e a Choctaw (Campagnolo \& Pio, 2012b).

A poda da amoreira-preta é realizada em dois momentos: no verão, quando eliminam-se as hastes que produziram e encurtam-se as novas hastes emergidas do solo na primavera; e no inverno, com redução das hastes laterais (Campagnolo \& Pio, 2012d). Uma alternativa seria adotar manejos de poda diferentes em regiões subtropicais, em que as podas drásticas possam ser realizadas, para eliminar todas as hastes rentes ao solo, sem prejuízos à produção. São necessários apenas cinco meses, a contar da emissão das hastes do solo, para que as gemas se diferenciem e estejam prontas para a brotação e a emissão de flores (Moore, 1984; Clark et al., 2005). A poda drástica de verão, realizada logo após o término da colheita, foi testada por Campagnolo \& Pio (2012c) na cultivar Tupy. Os autores verificaram que esse sistema de poda facilita a condução das plantas, sem prejudicar a qualidade dos frutos colhidos e o desempenho produtivo.
Ao se considerar a alta produtividade e a excelente qualidade dos frutos de outras cultivares, como a Brazos e a Guarani, e a Caingangue e a Choctaw, respectivamente, é preciso avaliar se esse manejo diferenciado de poda influencia a produção dessa frutífera, em regiões subtropicais.

SegundoAntunes (2002), a amoreira-preta éinfestada por algumas doenças, como antracnose [Elsinoë veneta (Burkh.) Jenkins, fase imperfeita Sphaceloma necator (Elis e Everh.)] e cercospora [Cercosporella rubi (G. Winter) Plakidas]. A antracnose incide principalmente em ramos, e a cercospora, em folhas, ambas com maior incidência no verão, quando as plantas estão em fase vegetativa. Acredita-se que a eliminação total das hastes após o período de colheita possa minimizar a infestação dessas doenças.

O objetivo deste trabalho foi avaliar o manejo de podas em cultivares de amoreira-preta, submetidas à poda drástica de verão, em regiões subtropicais.

\section{Material e Métodos}

O experimento foi conduzido no Município de Lavras, MG $\left(21^{\circ} 14^{\prime} \mathrm{S}, 45^{\circ} 00^{\prime} \mathrm{W}\right.$, a $918 \mathrm{~m}$ de altitude média), no período de janeiro de 2012 a janeiro de 2014. O clima da região é do tipo Cwb, mesotérmico ou tropical de altitude, com inverno seco e verão chuvoso, de acordo com a classificação de Köppen. As temperaturas médias máximas e mínimas e a precipitação acumulada de janeiro de 2012 a janeiro de 2014 estão descritos na Figura 1.

Utilizou-se o delineamento de blocos ao acaso, em arranjo fatorial $2 \times 7$ (podas $\mathrm{x}$ cultivares), em que o primeiro fator foi o uso de poda convencional (controle) e de poda drástica de verão, e o segundo foram as cultivares, com quatro blocos e 12 plantas úteis por unidade experimental.

Mudas das cultivares de amoreira-preta Comanche, Caingangue, Choctaw, Tupy, Guarani, Brazos e Cherokee foram produzidas com estacas radiculares, conforme a metodologia de Campagnolo \& Pio (2012a), e foram plantadas no campo em novembro de 2009, no espaçamento 3,0x0,5 m (densidade de 6.667 plantas por hectare). As plantas foram conduzidas sob espaldeira simples de um fio em "T" (fios duplos paralelos, espaçados a $60 \mathrm{~cm}$ de distância e a $80 \mathrm{~cm}$ de altura do solo). As plantas foram cultivadas seguindo as recomendações de Campagnolo \& Pio (2012d), 
para o cultivo de amoreiras em condições subtropicais. No entanto, não foram aplicados defensivos químicos para o controle de doenças. Durante a condução do experimento, foi realizado o controle das plantas espontâneas em toda a área experimental e efetuadas duas aplicações de adubo de compostagem (5 L por metro linear), uma em outubro e outra em junho, em cada ano de cultivo; o adubo foi uma mistura decomposta de material vegetal e esterco de curral na proporção $3: 1$. Nos dois anos de cultivo, realizouse a análise do solo. $\mathrm{O}$ solo apresentou as seguintes características: $\mathrm{pH}$ em água de 5,$7 ; 46,3 \mathrm{mg} \mathrm{dm}^{-3}$ de MO; 136,9 cmol $_{\mathrm{c}} \mathrm{dm}^{-3}$ de $\mathrm{P}$ (método Mehlich 1); $10,1 \mathrm{cmol}_{\mathrm{c}} \mathrm{dm}^{-3}$ de $\mathrm{Ca} ; 3,1 \mathrm{cmol}_{\mathrm{c}} \mathrm{dm}^{-3} \mathrm{de} \mathrm{Mg}$; saturação por bases de 74,2; e CTC de 15,5. Para a manutenção das plantas, foi aplicado $200 \mathrm{~g}$ de sulfato de amônio, divididos em duas aplicações, uma em setembro e outra em janeiro, além de $100 \mathrm{~g}$ de superfosfato simples, $100 \mathrm{~g}$ de cloreto de cálcio e $150 \mathrm{~g}$ de calcário por metro linear, aplicados em abril.

O manejo de podas foi executado de acordo com Campagnolo \& Pio (2012c). No tratamento controle (poda convencional), foram realizadas a poda drástica, rente ao solo, das hastes que produziram ao término da colheita, em 30 de janeiro, bem como a manutenção de quatro novas hastes primárias, com redução dos ápices por desponte. No inverno, no início de junho, foram feitas a redução das quatro hastes primárias a $30 \mathrm{~cm}$ acima do fio de arame da espaldeira e a manutenção de oito hastes secundárias, que foram reduzidas a $20 \mathrm{~cm}$ de comprimento. Na poda drástica de verão, realizouse a redução de todas as hastes a $5 \mathrm{~cm}$ do solo logo após o término da colheita, em 30 de janeiro. No início de junho, foram mantidas apenas quatro hastes por planta, a $30 \mathrm{~cm}$ acima do fio de arame da espaldeira, e feita a manutenção de oito hastes secundárias, a $20 \mathrm{~cm}$ de comprimento.

Passados 30 dias após a realização da poda drástica de verão, em janeiro de 2012 e 2013, foram determinados o número de rebentos (hastes) emitidos do solo e o comprimento médio desses aos 30 e 90 dias após a realização da poda.

Nos ciclos produtivos 2012/2013 e 2013/2014, foram marcados os estádios fenológicos: início, término e duração da florada e da colheita, a partir da poda realizada no início de junho até o encerramento

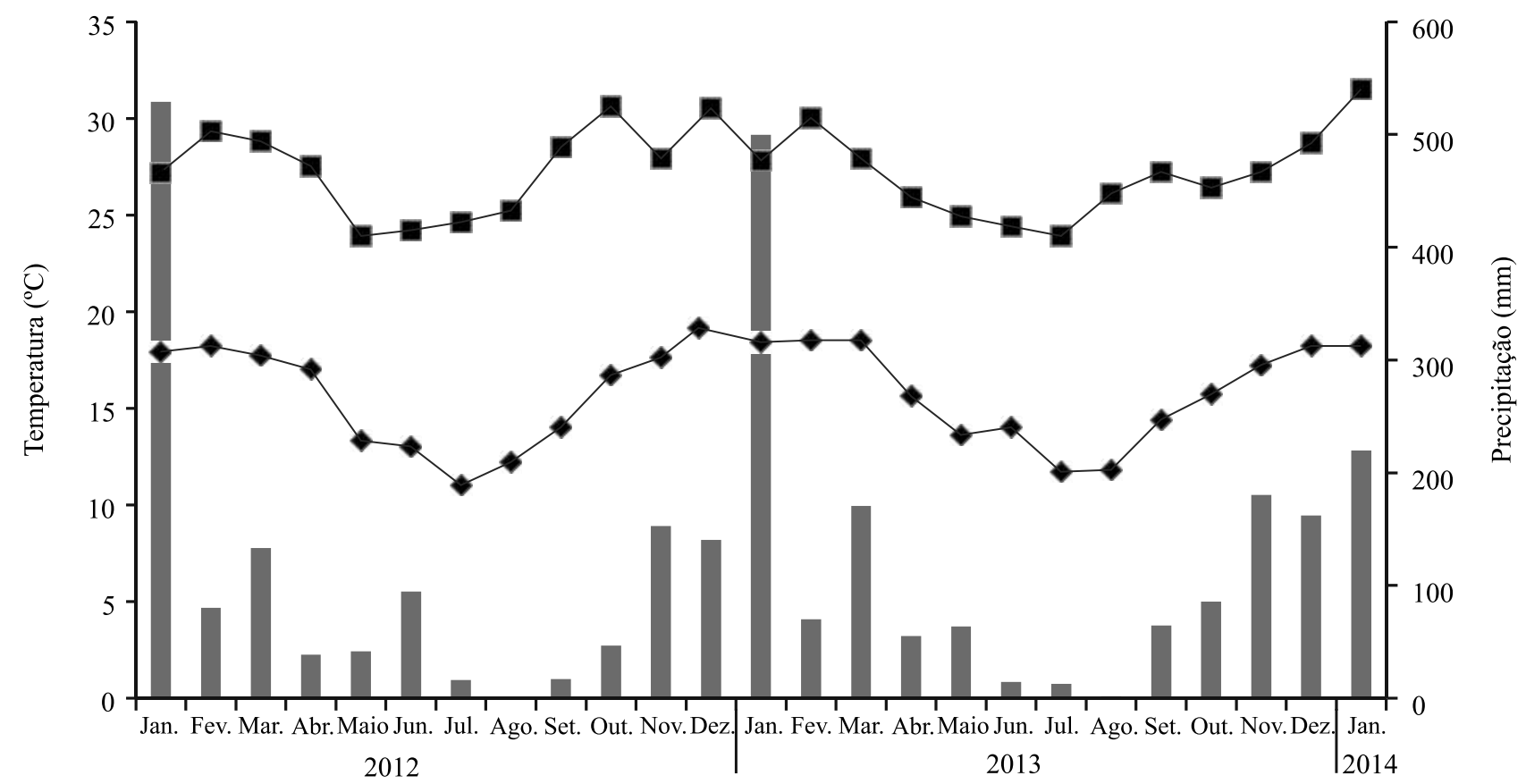

$\mathrm{T}^{\mathrm{o}}$ máx $\mathrm{T}^{\mathrm{o}}$ mín. Precipitação

Figura 1. Temperaturas médias máximas e mínimas e precipitação mensais de janeiro de 2012 a janeiro de 2014, em Lavras, MG. 
da colheita. As variáveis produtivas, como número de frutos por planta, produção (g por planta) e produtividade estimada $\left(\mathrm{Mg} \mathrm{ha}^{-1}\right)$, foram avaliadas de setembro a janeiro. Os frutos foram colhidos por parcela a cada três dias, contados e pesados com auxílio de balança semianalítica, modelo AUX220, (Shimadzu do Brazil, São Paulo, SP). Ao final do ciclo de produção, somaram-se todos os frutos e todas as massas registradas, para determinar a produção por planta e a produtividade estimada, por meio da multiplicação da produção pela densidade populacional (6.667 plantas por hectare). Durante o segundo ciclo produtivo, em dezembro, foram coletados 20 frutos por bloco para as determinações das características físico-químicas: comprimento e diâmetro médio dos frutos, com auxílio de paquímetro digital $150 \mathrm{~mm}$ (Kingtools, São Paulo, SP); massa média dos frutos, com balança semianalítica, modelo AUX220, (Shimadzu do Brasil, São Paulo, SP); acidez titulável (AT), obtida por meio da titulação das amostras com soluções de $\mathrm{NaOH} 0,1 \mathrm{~N}$, expressa em percentagem de ácido cítrico; e sólidos solúveis (SS), com auxílio de refratômetro portátil, modelo RTD-45, (Instrutherm Instrumentos de Medição Ltda., São Paulo, SP), a $20^{\circ} \mathrm{C}$, com leitura expressa em ${ }^{\circ}$ Brix e relação SS/AT.

Para a determinação da incidência da antracnose (Colletotrichum gloesporioides) em ramos das cultivares submetidas aos dois manejos de podas, verificou-se, em dez ramos de cada bloco, a presença ou a ausência de lesões localizadas entre o terço médio e a extremidade do ramo, para posterior cálculo da percentagem de incidência desse fungo. Quanto à cercosporiose (Cercosperella rubi), em razão de essa doença não ter sido relatada e descrita no Brasil para a amoreira-preta, foi montada uma escala diagramática do grau de infestação nas folhas, dividida em seis notas, conforme Smith \& Killebrew (2002): nota 0, folha sadia; nota 1,0 a $20 \%$ de área foliar lesionada; nota 2,20 a $40 \%$ de área foliar lesionada; nota 3, 40 a $60 \%$ de área foliar lesionada; nota 4,60 a $80 \%$ de área foliar lesionada; e nota 5, 80 a 100\% de área foliar lesionada. As folhas foram analisadas no terço médio dos ramos e na extremidade dos ramos. As análises das doenças foram realizadas no final do ciclo produtivo 2013/2014, em abril de 2014.

Os resultados obtidos foram submetidos à análise de variância, a 5\% de probabilidade, pelo teste de Scott \&
Knott, para comparações múltiplas. As análises foram realizadas com uso do programa Sisvar (Ferreira, 2011).

\section{Resultados e Discussão}

O início da floração das plantas ocorreu em meados de setembro, no primeiro ciclo produtivo, e em julho, no segundo ciclo, para a maioria das cultivares, à exceção da Comanche, que iniciou a floração em meados de agosto, e da Caingangue, no início de setembro. No primeiro ciclo, o término da floração ocorreu em janeiro, exceto para as cultivares Cherokee e Comanche, em que ocorreu em dezembro; no segundo ciclo, o encerramento da floração se deu em janeiro (Tabela 1). O florescimento provavelmente foi antecipado em decorrência do aumento das chuvas em março de 2013, seguido de um período de estiagem em junho (Figura 1).

Houve interação entre as cultivares e a poda convencional e a poda drástica das hastes no verão, para duração da floração e da colheita, nos dois ciclos de produção. O manejo de podas influenciou a duração do florescimento apenas nas cultivares Cherokee, no primeiro ciclo, e na Comanche em ambos os ciclos. Houve redução da duração da floração na cultivar Comanche e aumento na Cherokee, em plantas submetidas à poda drástica das hastes no verão. Entre as cultivares, a Caingangue permaneceu por menor tempo em floração (Tabela 1).

A alteração observada no início da floração, entre os ciclos produtivos, influenciou o início da colheita dos frutos. As colheitas iniciaram entre o final de setembro e o início de outubro e persistiram até janeiro, o que corrobora as observações de Campagnolo \& Pio (2012b), em experimento de competição de cultivares de amoreiras-pretas, em Marechal Cândido Rondon, PR, e de Segantini et al. (2011), em um trabalho de fenologia de plantas de amoreira-preta, em São Manoel, SP.

No primeiro ciclo produtivo, houve redução do período de colheita com a poda drástica de verão nas cultivares Brazos, Comanche e Caingangue, e, em ambos os ciclos, na cultivar Tupy, possivelmente em decorrência da homogeneização do crescimento dos rebentos (hastes) com a adoção da poda drástica de verão (Campagnolo \& Pio, 2012c). A cultivar Caingangue apresentou menor duração do período de colheita no segundo ciclo produtivo. 
Quanto ao desempenho da produção, houve interação entre as cultivares e a poda convencional e a poda drástica das hastes no verão. Plantas submetidas à poda drástica das hastes no verão produziram maior número de frutos, exceto a cultivar Comanche, no primeiro ciclo produtivo, e a Guarani, nos dois ciclos avaliados (Tabela 2). Assim, o mesmo resultado foi constatado na quantificação da produção por planta e na produtividade estimada.

Campagnolo \& Pio (2012c) observaram que plantas da cultivar Tupy submetidas à poda drástica das hastes no verão apresentaram maior desempenho produtivo do que as plantas que receberam poda convencional. No presente trabalho, as plantas submetidas à poda drástica das hastes no verão apresentaram incremento em produtividade, em comparação às que receberam podas convencionais, de $5,37 \mathrm{Mg} \mathrm{ha}^{-1}$ para a cultivar Brazos, de 1,51 Mg ha' $\mathrm{Mara}^{-1}$ a Tupy e de 1,01 $\mathrm{Mg} \mathrm{ha}^{-1}$ para a Comanche, no segundo ciclo produtivo.

$\mathrm{Na}$ poda convencional, mantêm-se apenas quatro hastes primárias por planta após a poda realizada ao final da colheita, e, na poda drástica de verão, as hastes emitidas crescem livremente. Dessa forma, o número de folhas é superior nas plantas que recebem a poda drástica de verão, em detrimento do maior número de hastes por planta, possivelmente porque há maior produção de fotoassimilados (reservas) e, consequentemente, maior emissão de flores (Campagnolo \& Pio, 2012c).

As cultivares diferiram quanto ao número de rebentos (hastes) emitidos e quanto ao crescimento das hastes. Na cultivar Brazos, houve emissão, em média, de oito rebentos por planta, que apresentaram rápido crescimento. Aos 30 dias após a realização da poda drástica das hastes, esses rebentos tinham em média $70 \mathrm{~cm}$ de comprimento e, aos 90 dias após a poda, mais de $170 \mathrm{~cm}$, nos dois anos consecutivos de avaliação. Outras cultivares, como a Guarani e a Tupy, também tiveram elevado número de rebentos emitidos (Tabela 3), o que afetou o comportamento produtivo das cultivares. O maior número de frutos por planta foi registrado para a cultivar Guarani, em ambos os ciclos produtivos avaliados, apesar de não ter havido diferença significativa no segundo ciclo em relação à cultivar Brazos (Tabela 2). A cultivar Brazos apresentou maior produção e produtividade estimada do que as cultivares Guarani e Tupy, que apresentam bom desempenho produtivo em regiões subtropicais

Tabela 1. Descrição fenológica dos ciclos produtivos 2012/2013 e 2013/2014 de cultivares de amoreira-preta (Rubus spp.) submetidas à poda convencional $(\mathrm{PC})$ e à poda drástica das hastes no verão $(\mathrm{PD})$, em Lavras, MG.

\begin{tabular}{|c|c|c|c|c|c|c|c|c|c|c|c|c|}
\hline \multirow[t]{2}{*}{ Cultivar } & \multicolumn{2}{|c|}{ IF } & \multicolumn{2}{|c|}{ TF } & \multicolumn{2}{|c|}{ DF $(\operatorname{dias})^{(1)}$} & \multicolumn{2}{|c|}{ IC } & \multicolumn{2}{|c|}{$\mathrm{TC}$} & \multicolumn{2}{|c|}{$\mathrm{DC}(\operatorname{dias})^{(1)}$} \\
\hline & $\mathrm{PD}$ & $\mathrm{PC}$ & PD & $\mathrm{PC}$ & $\mathrm{PD}$ & $\mathrm{PC}$ & $\mathrm{PD}$ & $\mathrm{PC}$ & $\mathrm{PD}$ & $\mathrm{PC}$ & $\mathrm{PD}$ & $\mathrm{PC}$ \\
\hline & \multicolumn{12}{|c|}{ Ciclo produtivo $2012 / 2013$} \\
\hline Guarani & $30 / 8$ & $30 / 8$ & $10 / 1$ & $10 / 1$ & $133 \mathrm{Aa}$ & $133 \mathrm{Aa}$ & $9 / 10$ & $9 / 10$ & $18 / 1$ & $20 / 1$ & $101 \mathrm{Aa}$ & $103 \mathrm{Ab}$ \\
\hline Tupy & $27 / 9$ & $15 / 9$ & $4 / 1$ & $1 / 1$ & 99Ac & $108 \mathrm{Ab}$ & $22 / 10$ & $11 / 10$ & $18 / 1$ & $20 / 1$ & $88 \mathrm{Bb}$ & $101 \mathrm{Ab}$ \\
\hline Brazos & $30 / 8$ & $30 / 8$ & $10 / 1$ & $10 / 1$ & $133 \mathrm{Aa}$ & $133 \mathrm{Aa}$ & $9 / 10$ & $9 / 10$ & $17 / 1$ & $28 / 1$ & $100 \mathrm{Ba}$ & $111 \mathrm{Aa}$ \\
\hline Cherokee & $4 / 9$ & $19 / 9$ & $1 / 1$ & $6 / 12$ & $119 \mathrm{Ab}$ & $78 \mathrm{Bc}$ & $11 / 10$ & $11 / 10$ & $18 / 1$ & $18 / 1$ & 99Aa & $99 \mathrm{Ab}$ \\
\hline Choctaw & $4 / 9$ & $4 / 9$ & $1 / 1$ & $4 / 1$ & $119 \mathrm{Ab}$ & $122 \mathrm{Ab}$ & $9 / 10$ & $9 / 10$ & $18 / 1$ & $22 / 1$ & $101 \mathrm{Aa}$ & $105 \mathrm{Ab}$ \\
\hline Comanche & $10 / 9$ & $27 / 8$ & $1 / 12$ & $22 / 12$ & $82 \mathrm{Bd}$ & $117 \mathrm{Ab}$ & $11 / 10$ & $9 / 10$ & $8 / 1$ & $20 / 1$ & $89 \mathrm{Bb}$ & $103 \mathrm{Ab}$ \\
\hline Caingangue & $24 / 10$ & $31 / 10$ & $8 / 1$ & $6 / 1$ & 76Ad & 67Ac & $29 / 10$ & $11 / 10$ & $18 / 1$ & $19 / 1$ & $81 \mathrm{Bc}$ & $100 \mathrm{Ab}$ \\
\hline \multirow[t]{2}{*}{ CV $(\%)$} & \multicolumn{2}{|c|}{-} & \multicolumn{2}{|c|}{-} & \multicolumn{2}{|c|}{8,10} & \multicolumn{2}{|c|}{-} & \multicolumn{2}{|c|}{-} & \multicolumn{2}{|c|}{3,20} \\
\hline & \multicolumn{12}{|c|}{ Ciclo produtivo $2013 / 2014$} \\
\hline Guarani & $11 / 7$ & $8 / 7$ & $7 / 1$ & $7 / 1$ & $180 \mathrm{Aa}$ & $184 \mathrm{Aa}$ & $30 / 9$ & $25 / 9$ & $30 / 1$ & $31 / 1$ & $122 \mathrm{Aa}$ & $128 \mathrm{Aa}$ \\
\hline Tupy & $9 / 7$ & $9 / 7$ & $15 / 1$ & $12 / 1$ & 190Aa & $187 \mathrm{Aa}$ & $18 / 11$ & $16 / 10$ & $29 / 1$ & $31 / 1$ & $72 \mathrm{Bb}$ & $107 \mathrm{Aa}$ \\
\hline Brazos & $8 / 7$ & $8 / 7$ & $7 / 1$ & $7 / 1$ & $183 \mathrm{Aa}$ & $183 \mathrm{Aa}$ & $25 / 9$ & $25 / 9$ & $25 / 1$ & $25 / 1$ & $122 \mathrm{Aa}$ & $122 \mathrm{Aa}$ \\
\hline Cherokee & $8 / 7$ & $11 / 7$ & $10 / 1$ & $7 / 1$ & $186 \mathrm{Aa}$ & $180 \mathrm{Aa}$ & $25 / 9$ & $3 / 10$ & $30 / 1$ & $30 / 1$ & $127 \mathrm{Aa}$ & $119 \mathrm{Aa}$ \\
\hline Choctaw & $15 / 7$ & $17 / 7$ & $12 / 1$ & $5 / 1$ & $181 \mathrm{Aa}$ & $172 \mathrm{Aa}$ & $25 / 9$ & $25 / 9$ & $31 / 1$ & $30 / 1$ & $128 \mathrm{Aa}$ & $127 \mathrm{Aa}$ \\
\hline Comanche & $13 / 8$ & $8 / 7$ & $7 / 1$ & $3 / 1$ & $147 \mathrm{Bb}$ & 179Aa & $30 / 9$ & $25 / 9$ & $31 / 1$ & $31 / 1$ & $123 \mathrm{Aa}$ & $128 \mathrm{Aa}$ \\
\hline Caingangue & $6 / 9$ & $6 / 9$ & $15 / 1$ & $15 / 1$ & $131 \mathrm{Ab}$ & $131 \mathrm{Ab}$ & $22 / 11$ & $22 / 11$ & $29 / 1$ & $31 / 1$ & $68 \mathrm{Ab}$ & $70 \mathrm{Ab}$ \\
\hline CV (\%) & \multicolumn{2}{|c|}{ - } & \multicolumn{2}{|c|}{-} & \multicolumn{2}{|c|}{8,19} & \multicolumn{2}{|c|}{-} & \multicolumn{2}{|c|}{-} & \multicolumn{2}{|c|}{11,79} \\
\hline
\end{tabular}


(Antunes et al., 2000; Campagnolo \& Pio, 2012c). Esse fato está relacionado à massa média dos frutos, pois a cultivar Brazos produz frutos de maior dimensão e massa (Tabela 4). O resultado do presente trabalho corrobora o encontrado por Antunes et al. (2000), que também registraram maior produtividade para a cultivar Brazos, seguida da Guarani e da Tupy, em Caldas, MG.

As cultivares que apresentaram menor produção foram a Cherokee e a Caingangue (Tabela 2). Campagnolo \& Pio (2012b) obtiveram praticamente as mesmas produtividades para as cultivares Comanche, Cherokee e Caingangue. Essas cultivares emitiram poucos rebentos após a poda drástica de verão e apresentaram menor crescimento das hastes (Tabela 3), o que pode estar associado à adaptação dessas cultivares ao clima subtropical (Campagnolo \& Pio, 2012b).

No segundo ciclo, a produção foi superior à registrada no primeiro. Em outubro e dezembro de 2012, as temperaturas máximas atingiram $30,5^{\circ} \mathrm{C}$ (Figura 1). Segundo Figueiredo et al. (2013), a temperatura ótima para a germinação dos grãos de pólen das amoreiras-pretas é de $27^{\circ} \mathrm{C}$. Assim, temperaturas elevadas podem ocasionar diminuição significativa na germinação dos grãos de pólen, o que afeta a produção de drupetes e a fixação de frutos e, portanto, diminui a produção das cultivares (Chagas et al., 2010).

Quanto às características físico-quimicas dos frutos produzidos, não houve diferença significativa entre os manejos de podas utilizados, apenas entre as cultivares. Campagnolo \& Pio (2012c) constataram que a poda drástica das hastes no verão não afetou a qualidade dos frutos da cultivar Tupy, em relação aos frutos dessa cultivar obtidos com a poda convencional.

Houve diferença significativa entre as cultivares quanto às características físico-químicas dos frutos produzidos. Frutos da cultivar Brazos apresentaram maiores dimensões e massa fresca, seguidos dos frutos das cultivares Tupy e Guarani, que não diferiram entre si (Tabela 4). Frutos das cultivares Choctaw e Caingangue apresentaram maior teor de sólidos solúveis, enquanto os da Cherokee e da Comanche apresentaram maior acidez titulável. Entretanto, a maior relação sólidos solúveis e acidez foi registrada em frutos da cultivar Caingangue, seguidos dos frutos das cultivares Tupy e Choctaw, o que confirma os

Tabela 2. Número médio de frutos, produção e produtividade estimada dos ciclos produtivos 2012/2013 e 2013/2014 de plantas de cultivares de amoreira-preta (Rubus spp.) submetidas à poda convencional e à poda drástica das hastes no verão, em Lavras, $\mathrm{MG}^{(1)}$.

\begin{tabular}{|c|c|c|c|c|c|c|}
\hline \multirow[t]{2}{*}{ Cultivar } & \multicolumn{2}{|c|}{ Número de frutos } & \multicolumn{2}{|c|}{ Produção por planta (g) } & \multicolumn{2}{|c|}{ Produtividade estimada $\left(\mathrm{Mg} \mathrm{ha}^{-1}\right)^{(2)}$} \\
\hline & Poda drástica & Poda convencional & Poda drástica & Poda convencional & Poda drástica & Poda convencional \\
\hline & \multicolumn{6}{|c|}{ Ciclo produtivo $2012 / 2013$} \\
\hline Guarani & $375,5 \mathrm{Aa}$ & $370,0 \mathrm{Aa}$ & $1.631,1 \mathrm{Ab}$ & $1.652,8 \mathrm{Aa}$ & $10,87 \mathrm{Ab}$ & $11,01 \mathrm{Aa}$ \\
\hline Tupy & $231,8 \mathrm{Ad}$ & $196,5 \mathrm{Bd}$ & $1.578,3 \mathrm{Ab}$ & $1.187,1 \mathrm{Bb}$ & $10,52 \mathrm{Ab}$ & $7,91 \mathrm{Bb}$ \\
\hline Brazos & $355,2 \mathrm{Ab}$ & $320,0 \mathrm{Bb}$ & $2.074,5 \mathrm{Aa}$ & $1.735,5 \mathrm{Ba}$ & $13,83 \mathrm{Aa}$ & $11,57 \mathrm{Ba}$ \\
\hline Cherokee & $127,0 \mathrm{Af}$ & $51,5 \mathrm{Bf}$ & $481,7 \mathrm{Ae}$ & $241,6 \mathrm{Bc}$ & $3,21 \mathrm{Ae}$ & $1,61 \mathrm{Be}$ \\
\hline Choctaw & $177,0 \mathrm{Ae}$ & $138,2 \mathrm{Be}$ & $713,7 \mathrm{Ad}$ & $496,3 \mathrm{Bc}$ & $4,75 \mathrm{Ad}$ & $3,31 \mathrm{Bc}$ \\
\hline Comanche & $305,7 \mathrm{Ac}$ & $296,0 \mathrm{Ac}$ & $1.170,9 \mathrm{Ac}$ & $1.112,8 \mathrm{Ab}$ & $7,81 \mathrm{Ac}$ & $7,42 \mathrm{Ab}$ \\
\hline Caingangue & $125,2 \mathrm{Af}$ & $96,3 \mathrm{Bf}$ & $390,8 \mathrm{Ae}$ & $366,7 \mathrm{Ac}$ & $2,61 \mathrm{Ae}$ & $2,44 \mathrm{Ad}$ \\
\hline \multirow[t]{2}{*}{$\mathrm{CV}(\%)$} & \multicolumn{2}{|c|}{12,38} & \multicolumn{2}{|c|}{14,28} & \multicolumn{2}{|c|}{14,28} \\
\hline & \multicolumn{6}{|c|}{ Ciclo produtivo $2013 / 2014$} \\
\hline Guarani & $390,2 \mathrm{Ab}$ & $385,2 \mathrm{Aa}$ & $1.691,9 \mathrm{Ab}$ & $1.671,8 \mathrm{Ab}$ & $11,28 \mathrm{Ab}$ & $11,14 \mathrm{Ab}$ \\
\hline Tupy & $275,7 \mathrm{Ac}$ & $259,7 \mathrm{Bb}$ & $1.754,3 \mathrm{Ab}$ & $1.528,2 \mathrm{Bb}$ & $11,69 \mathrm{Ab}$ & $10,18 \mathrm{Bb}$ \\
\hline Brazos & $451,2 \mathrm{Aa}$ & $384,7 \mathrm{Ba}$ & $2.749,8 \mathrm{Aa}$ & $1.944,2 \mathrm{Ba}$ & $18,33 \mathrm{Aa}$ & $12,96 \mathrm{Ba}$ \\
\hline Cherokee & $141,0 \mathrm{Ad}$ & $63,5 \mathrm{Bd}$ & $552,6 \mathrm{Ae}$ & $234,6 \mathrm{Be}$ & $3,68 \mathrm{Ae}$ & $1,56 \mathrm{Bf}$ \\
\hline Choctaw & $192,0 \mathrm{Ad}$ & $149,7 \mathrm{Bc}$ & $705,5 \mathrm{Ad}$ & $503,4 \mathrm{Bd}$ & $4,70 \mathrm{Ad}$ & $3,36 \mathrm{Bd}$ \\
\hline Comanche & $317,0 \mathrm{Ac}$ & $280,7 \mathrm{Bb}$ & $1.200,3 \mathrm{Ac}$ & $1.048,2 \mathrm{Bc}$ & $8,00 \mathrm{Ac}$ & $6,99 \mathrm{Bc}$ \\
\hline Caingangue & $150,7 \mathrm{Ad}$ & $83,7 \mathrm{Bd}$ & $499,5 \mathrm{Ae}$ & $366,2 \mathrm{Be}$ & $3,33 \mathrm{Ae}$ & $2,44 \mathrm{Be}$ \\
\hline $\mathrm{CV}(\%)$ & \multicolumn{2}{|c|}{14,44} & \multicolumn{2}{|c|}{15,61} & \multicolumn{2}{|c|}{15,61} \\
\hline
\end{tabular}

${ }^{(1)}$ Médias seguidas de letras iguais, maiúsculas nas linhas e minúsculas nas colunas, não diferem pelo teste de Scott \& Knott, a $5 \%$ de probabilidade. ${ }^{(2)}$ Calculado com espaçamento $3,0 \times 0,5 \mathrm{~m}$, com densidade de 6.667 plantas por hectare. 
resultados obtidos por Campagnolo \& Pio (2012b), em frutos dessas cultivares produzidos nas condições subtropicais do oeste do Estado do Paraná. Segundo Hirschi et al. (2012), a acidez e o teor de açúcar são dois importantes parâmetros utilizados como referência para classificar as polpas, para a produção de sucos ou para o mercado de fruta fresca.

Ressalta-se que podem ocorrer variações nos teores dos compostos químicos conforme o local onde as plantas são cultivadas, em razão das diferenças na intensidade de radiação solar e na amplitude térmica, que influenciam as características organolépticas dos frutos (Souza et al., 2014). Antunes et al. (2010) registraram $8,9^{\circ}$ Brix em frutos da cultivar Caingangue

Tabela 3. Número de rebentos (hastes) emitidos após 30 dias e comprimento médio das hastes aos 30 e 90 dias após a poda drástica de verão, nos ciclos vegetativos 2012 e 2013, de plantas de cultivares de amoreira-preta (Rubus spp.) submetidas à poda drástica das hastes no verão, em Lavras, $\mathrm{MG}^{(1)}$.

\begin{tabular}{lcccccccc}
\hline Cultivar & \multicolumn{2}{c}{$\begin{array}{c}\text { Número de } \\
\text { rebentos }\end{array}$} & & \multicolumn{3}{c}{ Comprimento das hastes } \\
\cline { 2 - 5 } \cline { 8 - 9 } & 2012 & 2013 & & 30 dias & 90 dias & & 30 dias & 90 dias \\
\hline Guarani & $7,0 \mathrm{~b}$ & $7,5 \mathrm{~b}$ & & $50,0 \mathrm{~b}$ & $140,0 \mathrm{~b}$ & & $59,1 \mathrm{~b}$ & $145,0 \mathrm{~b}$ \\
Tupy & $6,0 \mathrm{~b}$ & $6,2 \mathrm{~b}$ & & $41,0 \mathrm{c}$ & $152,0 \mathrm{~b}$ & & $54,6 \mathrm{~b}$ & $154,0 \mathrm{~b}$ \\
Brazos & $8,1 \mathrm{a}$ & $9,5 \mathrm{a}$ & & $68,5 \mathrm{a}$ & $176,2 \mathrm{a}$ & & $73,2 \mathrm{a}$ & $182,5 \mathrm{a}$ \\
Cherokee & $3,5 \mathrm{~d}$ & $4,0 \mathrm{~d}$ & & $43,7 \mathrm{c}$ & $93,5 \mathrm{c}$ & & $49,2 \mathrm{~b}$ & $125,7 \mathrm{c}$ \\
Choctaw & $5,2 \mathrm{c}$ & $4,2 \mathrm{c}$ & & $41,0 \mathrm{c}$ & $148,7 \mathrm{~b}$ & & $46,2 \mathrm{~b}$ & $130,7 \mathrm{c}$ \\
Comanche & $3,5 \mathrm{~d}$ & $4,7 \mathrm{c}$ & & $51,5 \mathrm{~b}$ & $130,0 \mathrm{~b}$ & & $53,0 \mathrm{~b}$ & $120,5 \mathrm{c}$ \\
Caingangue & $2,3 \mathrm{~d}$ & $2,3 \mathrm{~d}$ & & $38,3 \mathrm{c}$ & $103,3 \mathrm{c}$ & & $29,6 \mathrm{c}$ & $117,3 \mathrm{c}$ \\
\hline CV (\%) & 21,83 & 19,83 & & 14,63 & 17,32 & & 21,32 & 18,23 \\
\hline
\end{tabular}

${ }^{(1)}$ Médias seguidas de letras iguais não diferem pelo teste de Scott \& Knott, a $5 \%$ de probabilidade. produzidos em Pelotas, RS, isto é, $2,3^{\circ}$ Brix a menos do que os obtidos com a mesma cultivar no presente trabalho, em Lavras, MG.

Houve diferença nos manejos de podas quanto à incidência da antracnose em ramos apenas para as cultivares Choctaw e Comanche, que apresentaram a menor incidência dessa doença com uso da poda drástica de verão (Tabela 5). O elevado vigor e crescimento das cultivares Brazos e Guarani, e a alta incidência de antracnose, principalmente na Brazos, podem explicar porque o manejo de podas nessas duas cultivares não tenha sido eficiente na redução da antracnose. As cultivares Tupy e Cherokee apresentaram a menor incidência de antracnose em comparação às demais. Antunes et al. (2000) relatou que a cultivar Cherokee é moderadamente resistente à antracnose.

Embora a amoreira-preta apresente características de frutífera de clima temperado e hábito caducifólio, nas condições experimentais, não houve queda das folhas no período hibernal, apenas mudança na tonalidade das folhas, igualmente ao observado por Campagnolo \& Pio (2012c). Por esse motivo, os inóculos dos patógenos podem persistir nas plantas. A poda drástica de verão pode ser uma opção para a diminuição dos inóculos, pois remove o total das hastes no verão. Perdas de produção de frutas, como resultado do efeito de antracnose, têm sido entre 50 e $76 \%$, o que mostra a importância de melhorar o controle do patógeno (Rueda-Hernández et al., 2013).

Plantas das cultivares Tupy e Comanche, que receberam a poda drástica, apresentaram mais cercospora nas folhas no terço médio dos ramos (Tabela 5). Porém, na extremidade dos ramos, a incidência da doença foi menor.

Tabela 4. Características físico-químicas dos frutos oriundos do ciclo produtivo 2013/2014 de plantas de cultivares de amoreira-preta (Rubus spp.) submetidas à poda convencional e à poda drástica das hastes no verão, em Lavras, $\mathrm{MG}^{(1)}$.

\begin{tabular}{|c|c|c|c|c|c|c|}
\hline Cultivar & $\begin{array}{c}\text { Comprimento } \\
(\mathrm{mm})\end{array}$ & $\begin{array}{c}\text { Diâmetro } \\
(\mathrm{mm})\end{array}$ & $\begin{array}{c}\text { Massa fresca } \\
(\mathrm{g})\end{array}$ & $\begin{array}{c}\mathrm{SS}^{(2)} \\
\left({ }^{\circ} \text { Brix }\right) \\
\end{array}$ & $\begin{array}{c}\text { Acidez } \\
\left(\mathrm{g} 100 \mathrm{~g}^{-1}\right) \\
\end{array}$ & Relação SS/acidez \\
\hline Guarani & $23,86 b$ & $22,59 b$ & $7,94 b$ & $9,05 \mathrm{c}$ & $1,01 \mathrm{c}$ & $8,24 \mathrm{c}$ \\
\hline Tupy & $24,83 b$ & $22,13 b$ & $7,46 b$ & $10,15 b$ & $1,05 \mathrm{c}$ & $9,27 b$ \\
\hline Brazos & $27,86 a$ & $24,05 \mathrm{a}$ & $8,71 \mathrm{a}$ & $9,32 \mathrm{c}$ & $1,22 b$ & $7,78 \mathrm{~d}$ \\
\hline Cherokee & $19,24 \mathrm{c}$ & $18,61 \mathrm{c}$ & $4,49 d$ & $9,57 \mathrm{c}$ & $1,28 \mathrm{a}$ & $7,71 d$ \\
\hline Choctaw & $18,64 \mathrm{c}$ & $19,68 \mathrm{c}$ & $6,51 \mathrm{c}$ & $11,06 \mathrm{a}$ & $1,12 \mathrm{c}$ & $9,87 b$ \\
\hline Comanche & $20,30 \mathrm{c}$ & $19,69 \mathrm{c}$ & $5,54 d$ & $10,22 b$ & $1,28 \mathrm{a}$ & $8,29 \mathrm{c}$ \\
\hline Caingangue & $18,81 \mathrm{c}$ & $18,52 \mathrm{c}$ & $5,25 \mathrm{~d}$ & $11,52 \mathrm{a}$ & $1,16 b$ & $11,03 \mathrm{a}$ \\
\hline$\overline{\mathrm{CV}(\%)}$ & 6,89 & 7,42 & 7,76 & 5,93 & 10,45 & 9,56 \\
\hline
\end{tabular}

${ }^{(1)}$ Médias seguidas de letras iguais não diferem pelo teste de Scott \& Knott, a 5\% de probabilidade. ${ }^{(2)}$ SS, sólidos solúveis. 
Tabela 5. Incidência de antracnose (Colletotrichum gloesporioides) em ramos e escala de notas para cercospora (Cercosporella rubi) em folhas localizadas no terço médio e na extremidade dos ramos, no ciclo produtivo 2013/2014, de plantas de cultivares de amoreira-preta (Rubus spp.) submetidas à poda convencional e à poda drástica das hastes no verão, em Lavras, MG.

\begin{tabular}{|c|c|c|c|c|c|c|}
\hline \multirow[t]{2}{*}{ Cultivar } & \multicolumn{2}{|c|}{$\begin{array}{l}\text { Incidência de antracnose } \\
\text { em ramos }(\%)^{(1)}\end{array}$} & \multicolumn{2}{|c|}{$\begin{array}{l}\text { Escala de notas para cercospora em } \\
\text { folhas no terço médio dos ramos }\end{array}$} & \multicolumn{2}{|c|}{$\begin{array}{l}\text { Escala de notas para cercospora em } \\
\text { folhas na extremidade dos ramos }\end{array}$} \\
\hline & Poda drástica & Poda convencional & Poda drástica & Poda convencional & Poda drástica & Poda convencional \\
\hline Guarani & $42,5 \mathrm{Ab}$ & $52,5 \mathrm{Ab}$ & $1,77 \mathrm{Ac}$ & $2,15 \mathrm{Ab}$ & $0,82^{\text {ns }}$ & 0,65 \\
\hline Tupy & $2,5 \mathrm{Ac}$ & $10,0 \mathrm{Ad}$ & $3,22 \mathrm{Ab}$ & $2,35 \mathrm{Bb}$ & 1,82 & 1,27 \\
\hline Brazos & $92,5 \mathrm{Aa}$ & $90,0 \mathrm{Aa}$ & $2,35 \mathrm{Ab}$ & $1,90 \mathrm{Ab}$ & 1,25 & 0,97 \\
\hline Cherokee & $12,5 \mathrm{Ac}$ & 7,5Ad & $2,25 \mathrm{Ab}$ & $1,57 \mathrm{Ab}$ & 0,77 & 0,27 \\
\hline Choctaw & $45,0 \mathrm{Bb}$ & $62,5 \mathrm{Ab}$ & $2,77 \mathrm{Ab}$ & $2,12 \mathrm{Ab}$ & 0,87 & 0,75 \\
\hline Comanche & $42,5 \mathrm{Bb}$ & $77,5 \mathrm{Aa}$ & $3,47 \mathrm{Aa}$ & $2,87 \mathrm{Ba}$ & 1,20 & 1,02 \\
\hline Caingangue & $42,0 \mathrm{Ab}$ & $33,3 \mathrm{Ac}$ & 3,80Aa & $3,16 \mathrm{Aa}$ & 1,52 & 0,90 \\
\hline $\mathrm{CV}(\%)$ & \multicolumn{2}{|c|}{28,03} & \multicolumn{2}{|c|}{21,13} & \multicolumn{2}{|c|}{20,66} \\
\hline
\end{tabular}

${ }^{(1)}$ Médias seguidas de letras iguais, maiúsculas nas linhas e minúsculas nas colunas, não diferem pelo teste de Scott \& Knott, a $5 \%$ de probabilidade. ${ }^{\text {ns }}$ Não significativo.

\section{Conclusões}

1. A poda drástica de verão aumenta a produção de cultivares de amoreira-preta (Rubus spp.) em regiões subtropicais.

2. Não há diferença na qualidade dos frutos de amoreira-preta colhidos de plantas submetidas aos sistemas de poda convencional e poda drástica de verão.

3. A poda drástica de verão não é eficiente na redução ou no controle de doenças da amoreira-preta.

\section{Agradecimentos}

À Coordenação de Aperfeiçoamento de Pessoal de Nível Superior (Capes) e à Fundação de Amparo à Pesquisa do Estado de Minas Gerais (Fapemig), pelo apoio financeiro e pela concessão de bolsa de estudos.

\section{Referências}

ANTUNES, L.E.C. Amora-preta: nova opção de cultivo no Brasil. Ciência Rural, v.32, p.151-158, 2002. DOI: 10.1590/ S0103-84782002000100026.

ANTUNES, L.E.C.; CHALFUN, N.N.J.; REGINA, M.A.; DUARTE FILHO, J. Fenologia e produção de variedades de amora-preta nas condições do Planalto de Poços de Caldas-MG. Revista Brasileira de Fruticultura, v.22, p.89-95, 2000.

ANTUNES, L.E.C.; GONÇALVES, E.D.; TREVISAN, R. Fenologia e produção de cultivares de amoreira-preta em sistema agroecológico. Ciência Rural, v.40, p.1929-1933, 2010. DOI: 10.1590/S0103-84782010000900012.

BROETTO, D.; BOTELHO, R.V.; PAVANELlO, A.P.; SANTOS R.P. Cultivo orgânico de amora-preta cv. Xavante em Guarapuava-PR. Revista Brasileira de Agroecologia, v.4, p.2208-2212, 2009.

CAMPAGNOLO, M.A.; PIO, R. Enraizamento de estacas caulinares e radiculares de cultivares de amoreira-preta coletadas em diferentes épocas, armazenadas a frio e tratadas com AIB. Ciência Rural, v.42, p.232-237, 2012d. DOI: 10.1590/ S0103-84782012000200008.

CAMPAGNOLO, M.A.; PIO, R. Phenological and yield performance of black and redberry cultivars in western Paraná State. Acta Scientiarum. Agronomy, v.34, p.439-444, 2012a. DOI: 10.4025 /actasciagron.v34i4.15528.

CAMPAGNOLO, M.A.; PIO, R. Poda drástica para a produção da amora-preta em regiões subtropicais. Pesquisa Agropecuária Brasileira, v.47, p.934-938, 2012c. DOI: 10.1590/ S0100-204X2012000700009.

CAMPAGNOLO, M.A.; PIO, R. Produção da amoreira-preta 'Tupy' sob diferentes épocas de poda. Ciência Rural, v.42, p.225-231, 2012b. DOI: 10.1590/S0103-84782012005000007.

CHAGAS, E.A.; PIO, R.; CHAGAS, P.C.; PASQUAL, M.; BETTIOL NETO, J.E. Composição do meio de cultura e condições ambientais para germinação de grãos de pólen de porta-enxertos de pereira. Ciência Rural, v.40, p.261-266, 2010. DOI: 10.1590/ S0103-84782010000200002.

CLARK, J.R.; MOORE, J.N.; LOPEZ-MEDINA, J.; FINN, C.; PERKINS-VEAZIE, P. 'Prime-Jan' ('APF 8') and 'Prime-Jim' ('APF 12') primocane-fruiting blackberries. Hortscience, v.40, p.852-855, 2005.

FACHINELLO, J.C.; PASA, M. da S.; SCHMTIZ, J.L.; BETEMPS, D.L. Situação e perspectivas da fruticultura de clima temperado no Brasil. Revista Brasileira de Fruticultura, v.33, p.92-108, 2011. DOI: 10.1590/S0100-29452011000500014.

FERREIRA, D.F. Sisvar: a computer statistical analysis system. Ciência e Agrotecnologia, v.35, p.1039-1042, 2011. DOI: 10.1590/S1413-70542011000600001. 
FIGUEIREDO, M.A. de; PIO, R.; SILVA, T.C.; SILVA, K.N. Características florais e carpométricas e germinação in vitro de grãos de pólen de cultivares de amoreira-preta. Pesquisa Agropecuária Brasileira, v.48, p.731-740, 2013. DOI: 10.1590/ S0100-204X2013000700005.

GUEDES, M.N.S.; ABREU, C.M.P. de; MARO, L.A.C.; PIO, R.; ABREU, J.R.A.; OLIVEIRA, J.O. Chemical characterization and mineral levels in the fruits of blackberry cultivars grown in a tropical climate at an elevation. Acta Scientiarum. Agronomy, v.35, p.191-196, 2013. DOI: 10.4025/actasciagron.v35i2.16630.

HIRSCHI, G.E.; FACCO, E.M.P.; RODRIGUES, D.B.; VIZZOTTO, M.; EMANUELLI, T. Caracterização físico-química de variedades de amora-preta da região Sul do Brasil. Ciência Rural, v.42, p.942-947, 2012. DOI: 10.1590/S0103-84782012005000021.

MOORE, J.N. Blackberry breeding. HortScience, v.19, p.183-185, 1984.

RUEDA-HERNÁNDEZ, K.R.; CARDONA, A.S.; CADAVID-RESTREPO, G.E.; BENJUMEA, C.I.S.; GUTIERREZ,
G.P.C.; ISAZA, R.E.A.Differential organ distribution, pathogenicity and benomyl sensitivity of Colletotrichum spp. from blackberry plants in Northern Colombia. Journal of Phytopathology, v.161, p.246-253, 2013. DOI: 10.1111/jph. 12040.

SEGANTINI, D.M.; LEONEL, S.; RIPARDO, A.K. da S.; AURICCHIO, M.G.R. Uso de reguladores de crescimento para a superação da dormência e sua influência na brotação, no florescimento e na produção da amoreira-preta. Revista Brasileira de Fruticultura, v.33, p.275-280, 2011. DOI: 10.1590/S0100-29452011000500034.

SMITH, B.J.; KILLEBREW, J.F. Epidemiology and control of blackberry rosette caused by the fungus Cercosporella rubi. Acta Horticulturae, v.585, p.319-323, 2002.

SOUZA, V.R. de; PEREIRA, P.A.P.; SILVA, T.L.T. da; LIMA, L.C. de O.; PIO, R.; QUEIROZ, F. Determination of the bioactive compounds, antioxidant activity and chemical composition of Brazilian blackberry, red raspberry, strawberry, blueberry and sweet cherry fruits. Food Chemistry, v.156, p.362-368, 2014. DOI: 10.1016/j.foodchem.2014.01.125.

Recebido em 17 de junho de 2014 e aprovado em 31 de dezembro de 2014 